\title{
Magnetotransport in a time-modulated double quantum point contact system
}

\author{
Chi-Shung Tang ${ }^{*, a}$, Kristinn Torfason $^{\mathrm{b}}$, Vidar Gudmundsson ${ }^{\mathrm{b}}$ \\ ${ }^{a}$ Department of Mechanical Engineering, National United University, Miaoli 36003, Taiwan \\ ${ }^{b}$ Science Institute, University of Iceland, Dunhaga 3, IS-107 Reykjavik, Iceland
}

\begin{abstract}
We report on a time-dependent Lippmann-Schwinger scattering theory that allows us to study the transport spectroscopy in a timemodulated double quantum point contact system in the presence of a perpendicular magnetic field. Magnetotransport properties involving inter-subband and inter-sideband transitions are tunable by adjusting the time-modulated split-gates and the applied magnetic field. The observed magnetic field induced Fano resonance feature may be useful for the application of quantum switching.
\end{abstract}

Key words: magnetotransport, time-modulated, quantum point contact, magnetic field

\section{Introduction}

Electron transport in mesoscale devices smaller than the electron phase coherence length has received extensive studies [1]. Magnetotransport and time-dependent transport in gatecontrolled semiconducting systems are essential fundamental entities in mesoscopic physics. Recently, It was reported that the conductance involving Aharonov-Bohm (AB) interference as a function of magnetic field exhibits step-like structures [2]. Sigrit et al. measured the differential conductance of an AB interferometer by varying the bias voltage [3]. Their results indicate that varying either the magnetic field or the electrostatic confining potentials allows the interference to be tuned. In this work, we investigate the magneto-conductance in a double quantum point contact (DQPC) system by controlling two pairs of split-gate (SG) voltages for the manipulation of the dynamical electronic transport properties in the DQPC-confined cavity region.

\section{Model}

The system under investigation is supposed to be a parabolically confined quantum channel fabricated from a modulationdoped GaAs-based heterostructure with two-pairs of spit-gates defining the DQPC system treated as a scattering potential $V_{\mathrm{sc}}(x, y, t)$, as depicted in Fig. 1] The Hamiltonian describing the system can be expressed in the form

$$
\mathcal{H}(t)=-\frac{\hbar^{2}}{2 m^{*}}\left(\nabla^{2}-\frac{2 i}{l^{2}} y \partial_{x}-\frac{y^{2}}{l^{4}}\right)+\frac{m^{*}}{2} \Omega_{0}^{2} y^{2}+V_{\mathrm{sc}},
$$

in which the effective mass $m^{*}=0.067 m_{e}$, and the magnetic length $l=\hbar /(e B)$ is related to the perpendicular magnetic field $\mathbf{B}=B \hat{\mathbf{z}}$. The characteristic confining energy $\hbar \Omega_{0}$ of the parabolic

\footnotetext{
${ }^{*}$ Corresponding author

Email address: cstang@nuu.edu.tw (Chi-Shung Tang)
}

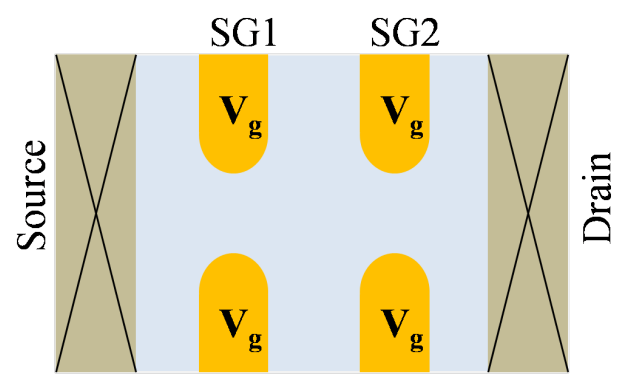

Figure 1: Schematic illustration of the double quantum point contact system constructed by two pairs of split-gates with gate voltages $V_{g}$ described by $V_{\mathrm{sc}}$ in our model.

confinement is modified by the applied magnetic field leading to the effective confining energy $\hbar \Omega_{\omega}=\hbar\left(\omega_{c}^{2}+\Omega_{0}^{2}\right)^{1 / 2}$ where $\omega_{\mathrm{c}}=e B /\left(m^{*} c\right)$. The scattering potential

$$
V_{\mathrm{sc}}(x, y, t)=V_{s}(x, y)+\sum_{i=1}^{2} V_{t}(x, y) \cos \left(\omega t+\phi_{i}\right)
$$

contains a static part $V_{s}$ as well as a time-dependent part with strength $V_{t}$ and driving frequency $\omega$. The time-modulated SGs may have an arbitrary phase $\phi_{i}$.

We employ the mixed momentum-coordinate representation [4] to transform the total wave function $\Psi(x, y, t)=$ $\sum_{n} \phi_{n}(y, p) \psi_{n}(p, t)$ into the wave function $\Psi(p, y, t)$ in terms of the eigenfunctions $\phi_{n}(y, p)$ of the unperturbed quantum channel. Performing the expansion allows us to obtain a coupled nonlocal time-dependent integral equation in the momentum space:

$$
\begin{aligned}
i \hbar \partial_{t} \psi_{n}(p, t)= & {\left[E_{n}(0)+K(p)\right] \psi_{n}(p, t) } \\
& +\sum_{n^{\prime}} \int \frac{q}{2 \pi} V_{n, n^{\prime}}(p, q, t) \psi_{n^{\prime}}(q, t) .
\end{aligned}
$$

This equation describes the electron propagation of an asymptotic state occupying subband $n$ along the $x$-direction from 
the source electrode. Here the subband threshold $E_{n}(0)=$ $(n+1 / 2) \hbar \Omega_{\omega}$ is determined by the lateral confinement and the effective kinetic energy $K(p)=\hbar^{2} p^{2}\left(\hbar \Omega_{0}\right)^{2} /\left[2 m^{*}\left(\hbar \Omega_{\omega}\right)^{2}\right]$. The matrix elements of the scattering potential

$$
V_{n, n^{\prime}}(p, q, t)=\int d y d x e^{-i(p-q) x} \phi_{n}^{*}(y, p) V(x, y, t) \phi_{n^{\prime}}(y, q)
$$

indicates the electrons in the subband $n$ may be making intersubband transitions to the intermediate subband $n^{\prime}$.

To proceed, it is convenient to transform the time-dependent wave function from the time domain to the frequency domain

$$
\psi_{n}(p, t)=\sum_{m=-\infty}^{\infty} e^{-i E_{m} t / \hbar} \psi_{n}^{m}(p)
$$

where the quasi-energy $E_{m}=E_{0}+m \hbar \omega$. Similarly, we have $V_{n, n^{\prime}}(p, q, t)=\sum_{m^{\prime}=-\infty}^{\infty} e^{-i m^{\prime} \omega t} V_{n n^{\prime}}^{m^{\prime}}(p, q)$ with $m^{\prime}$ indicating the photon sideband index. Defining the wave number of an electron occupying the subband $n$ and the sideband $m$ intermediate state

$$
\frac{1}{2}\left(\frac{k_{n}^{m}}{\beta}\right)^{2} \frac{\left(\hbar \Omega_{0}\right)^{2}}{\hbar \Omega_{\omega}}=E_{m}-E_{n}(0)
$$

and

$$
\widetilde{V}_{n, n^{\prime}}^{m-m^{\prime}}(q, p) \equiv 2 \frac{\left(\hbar \Omega_{\omega}\right)^{2}}{\left(\hbar \Omega_{0}\right)^{2}} \frac{\beta}{\hbar \Omega_{\omega}} V_{n, n^{\prime}}^{m-m^{\prime}}(q, p)
$$

allows us to obtain the multiple scattering identity

$$
\psi_{n}^{m}(q)=\left[\left(\frac{k_{n}^{m}}{\beta}\right)^{2}-\left(\frac{q}{\beta}\right)^{2}\right]^{-1} \sum_{m^{\prime} n^{\prime}} \int \frac{d p}{2 \pi} \widetilde{V}_{n, n^{\prime}}^{m-m^{\prime}}(q, p) \psi_{n^{\prime}}^{m^{\prime}}(p) .
$$

Taking all the intermediate states $\left(n^{\prime}, m^{\prime}\right)$ into account, we can obtain the Lippmann-Schwinger equation in the momentum space

$$
\begin{aligned}
\psi_{n}^{m}(q)= & \psi_{n}^{m, 0}(q)+G_{n}^{m}(q) \\
& \times \frac{1}{2 \pi} \sum_{n^{\prime}, m^{\prime}} \int d\left(\frac{p}{\beta}\right) \widetilde{V}_{n, n^{\prime}}^{m-m^{\prime}}(q, p) \psi_{n^{\prime}}^{m^{\prime}}(p)
\end{aligned}
$$

in terms of the unperturbed Green function $G_{n}^{m}(q)$. Since the incident wave $\psi_{n}^{m, 0}(q)$ is of the delta-function type, to achieve exact numerical computation one has to define the $T$ matrix

$$
\begin{aligned}
T_{n^{\prime}, n}^{m^{\prime}, m}(q, p) & =V_{n^{\prime}, n}^{m^{\prime}-m}(q, p) \\
& +\sum_{r, s} \int \frac{d k}{2 \pi} V_{n^{\prime}, r}^{m^{\prime}-s}(q, k) G_{r}^{s}(k) T_{r, n}^{s, m}(k, p)
\end{aligned}
$$

that couples all the intermediate states $(r, s)$. The potential is expanded in the Fourier series yields a connection between the sidebands for constructing the $T$ matrix

$$
\begin{aligned}
& T_{n^{\prime}, n}^{m^{\prime}, m}(q, p)=V_{s, n^{\prime} n}(q, p) \delta_{m^{\prime}-m, 0} \\
& +\frac{1}{2} V_{t, n^{\prime} n}(q, p)\left(\delta_{m^{\prime}-m,-1}+\delta_{m^{\prime}-m, 1}\right) \\
& +\sum_{r} \int \frac{d k}{2 \pi} V_{s, n^{\prime} r}(q, k) G_{r}^{m^{\prime}}(k) T_{r, n}^{m^{\prime}, m}(k, p) \\
& +\frac{1}{2} \sum_{r} \int \frac{d k}{2 \pi} V_{t, n^{\prime} r}^{+}(q, k) G_{r}^{m^{\prime}+1}(k) T_{r, n}^{\left(m^{\prime}+1\right), m}(k, p) \\
& +\frac{1}{2} \sum_{r} \int \frac{d k}{2 \pi} V_{t, n^{\prime} r}^{-}(q, k) G_{r}^{m^{\prime}-1}(k) T_{r, n}^{\left(m^{\prime}-1\right), m}(k, p)
\end{aligned}
$$

where $V_{t, n^{\prime} r}^{ \pm}(q, k)=\sum_{i} V_{t, n^{\prime} r}(q, k) e^{ \pm i \phi_{i}}$ coupling the adjacent sidebands. In terms of the $T$ matrix, we can obtain the momentum-space wave function

$$
\begin{aligned}
\psi_{n^{\prime}}^{m^{\prime}}(q)= & \psi_{n^{\prime}}^{m^{\prime}, 0}(q)+G_{n^{\prime}}^{m^{\prime}}(q) \\
& \times \sum_{n, m} \int \frac{d k}{2 \pi} T_{n^{\prime}, n}^{m^{\prime}, m}(q, k) \psi_{n}^{m, 0}(k) .
\end{aligned}
$$

Performing the inverse Fourier transform to the real space and the residue integration allows us to obtain the transmission amplitude of the electron wave along the $\underline{x}$-direction

$$
\underline{\mathrm{t}}_{n^{\prime}, n}^{m^{\prime}, 0}=\delta_{n^{\prime}, n} \delta_{m^{\prime}, 0}-\frac{i}{2 k_{n^{\prime}}^{m^{\prime}}} T_{n^{\prime}, n}^{m^{\prime}, 0}\left(k_{n^{\prime}}^{m^{\prime}}, k_{n}^{0}\right) .
$$

The time-average conductance can be obtained based on the Landauer-Büttiker framework [5, 6]

$$
G=G_{0} \sum_{m^{\prime}=-\infty}^{\infty} \operatorname{Tr}\left[\underline{t}_{n^{\prime}, n}^{m^{\prime}, 0}\left(\underline{\mathrm{t}}_{n^{\prime}, n}^{m^{\prime}, 0}\right)^{*}\right]
$$

with $G_{0}=2 e^{2} / h$. This indicates that the transmission matrix connecting the contribution from all the photon sideband $m^{\prime}$ of propagating modes has to be taken into account for the electrons occupying arbitrary subbands below the Fermi energy.

\section{Numerical Results}

We assume that the system is fabricated in a high-mobility GaAs-based heterostructure such that the effective Rydberg energy $E_{\mathrm{Ryd}} \approx 5.9 \mathrm{meV}$ and the Bohr radius $a_{\mathrm{B}} \approx 9.8 \mathrm{~nm}$. The confining parameter of the quantum channel is $\hbar \Omega_{0}=1 \mathrm{meV}$, the length is scaled by $\beta_{0}^{-1} \approx 33.7 \mathrm{~nm}$, and the energy is either in meV or in units of $\hbar \Omega_{\omega}$. The $\hbar \Omega_{\omega}=1.0148 \mathrm{meV}$ for the magnetic field $B=0.1 \mathrm{~T}$.

The time-modulated DQPC system is described by the scattering potential

$$
V_{\mathrm{sc}}(\underline{\mathrm{r}}, t)=V_{\mathrm{SG} 1}(\underline{\mathrm{r}}, t)+V_{\mathrm{SG} 2}(\underline{\mathrm{r}}, t)
$$

where

$$
V_{\mathrm{SG} 1}=V_{1}(t)\left[e^{-\alpha_{x}\left(x+x_{0}\right)^{2}}+e^{-\alpha_{x}\left(x+x_{0}\right)^{2}}\right] e^{-\alpha_{y}\left(y+y_{0}\right)^{2}}
$$

and

$$
V_{\mathrm{SG} 2}=V_{2}(t)\left[e^{-\alpha_{x}\left(x-x_{0}\right)^{2}}+e^{-\alpha_{x}\left(x-x_{0}\right)^{2}}\right] e^{-\alpha_{y}\left(y+y_{0}\right)^{2}}
$$

with $V_{i}(t)=V_{s}+V_{t} \cos \left(\omega t+\phi_{i}\right)$ and $i=1,2$. Moreover, we select $\left(\alpha_{x}, \alpha_{y}\right)=(0.5,0.3) \beta_{0}^{2}$, and $\left(x_{0}, y_{0}\right)=(8,3) \beta_{0}$ such that the gate-width $\sim 80 \mathrm{~nm}$ and the SG-confined cavity area $~$ $540 \times 200 \mathrm{~nm}^{2}$.

In Fig. 2, we show the conductance as a function of incident energy for the time-modulated DQPC with applied magnetic field $B=0.1 \mathrm{~T}$ (blue solid) in comparison with the zero magnetic field situation (red dashed). The DQPC system is confined by $V_{s}=6.0 \mathrm{meV}$, and the time-modulation with strength $V_{t}=1.5 \mathrm{meV}$ and frequency $\omega=0.17 \Omega_{\omega}$. In addition, we have assumed that the phase difference between the two split-gates 


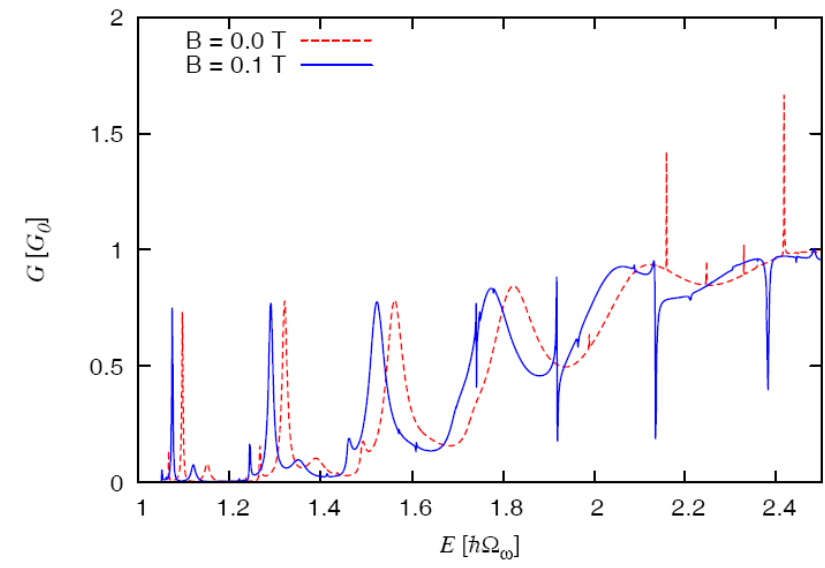

Figure 2: Conductance as a function of incident energy for the cases of $B=$ $0.0 \mathrm{~T}$ (red dashed) and $B=0.1 \mathrm{~T}$ (blue solid). The other parameters are $V_{s}=$ $6.0 \mathrm{meV}, V_{t}=1.5 \mathrm{meV}, \phi=\pi$, and $\omega=0.17 \Omega_{\omega}$.
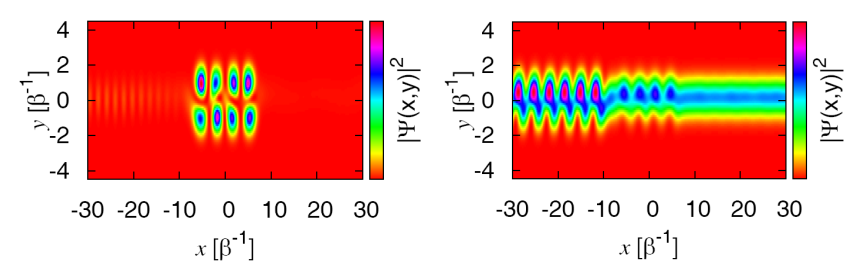

Figure 3: Probability density with magnetic field $B=0.1 \mathrm{~T}$ at the Fano peak with electronic energy $E / \hbar \Omega_{\omega}=1.918$ for the electron incident from the subband $n=0$ (left) and $n=1$ (right).

SG1 and SG2 is $\phi=\phi_{1}-\phi_{2}=\pi$. In general, the electron kinetic energy turns out to play a role of suppressing the quasibound state feature, namely suppressing the side-peak structures beneath a main resonance peak in conductance. However, in the high kinetic energy regime, an appropriate magnetic field may induce the time-modulated Fano antiresonance features at energies $E / \hbar \Omega_{\omega} \approx 1.75,1.92,2.16$ as well as the time-modulated Breit-Winger dip feature at $E / \hbar \Omega_{\omega} \approx 2.38$. Below, we focus on the robust Fano line-shape feature: The Fano-peak is at $E / \hbar \Omega_{\omega}=1.918$ and the Fano-dip is at $E / \hbar \Omega_{\omega}=1.920$, as depicted in Fig. 2 .

In order to get better understanding of the magnetic-field induced time-nodulated Fano antiresonance feature, we explore the electronic probability density with energies around the Fano line-shape. It is clearly shown in Fig. 3 that the electron occupying the first subband with higher kinetic energy favors to form a long-lived $(4,2)$ localized bound state in the cavity formed by the DQPC system. However, the electron occupying the second subband with lower kinetic energy is not fitting to the characteristic energies in the cavity and hence forming a short-lived $(4,1)$ extended state. The interference of the $n=0$ localized state and the $n=1$ extended state induces the Fano peak at the energy $E / \hbar \Omega_{\omega}=1.918$.

The probability density features for the electron with incident energy at $E / \hbar \Omega_{\omega}=1.920$ are demonstrated in Fig. 4. The electrons occupying the lowest subband $(n=0)$ can also form a long-lived $(4,2)$ localized state, but with higher coupling
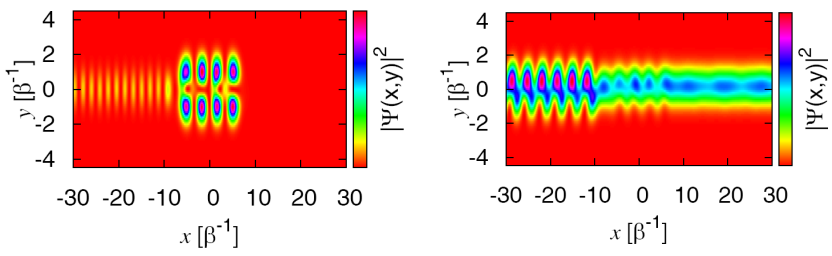

Figure 4: Probability density with magnetic field $B=0.1 \mathrm{~T}$ at the Fano dip with electronic energy $E / \hbar \Omega_{\omega}=1.920$ for the electron incident from the subband $n=0$ (left) and $n=1$ (right).

to source-lead thus forming the Fano-dip line-shape. For the electron occupying the second subband $(n=1)$ at $E / \hbar \Omega_{\omega}=$ 1.920 , the extended $(4,1)$ state is weaker than the electron with $E / \hbar \Omega_{\omega}=1.918$. The energy difference $\delta E_{\text {Fano }} \approx 2.03 \mu \mathrm{eV}$ between the Fano-peak and the Fano-dip should be within the observable resolution via the current transport measurement technique.

\section{Summary}

In summary, we have presented coherent magnetotransport numerical calculation on a time-modulated double QPC system and demonstrated dynamical control of the magnetic-field induced Fano interference by manipulating the applied magnetic field. It was reported that the anti-symmetric ac split-gate voltage can be utilized to induce the Fano resonance [7]. Differently, we have reported here by tuning an appropriate magnetic field in the DQPC system with symmetric ac split-gates to induce the Fano resonance that becomes non-resonant by switching off the applied magnetic field. This robust magnetic field induced dynamic Fano resonance feature may be useful for the magneto-control of quantum switching in arbitrary timemodulated mesoscopic systems.

\section{Acknowledgments}

This work was supported by the Research and Instruments Funds of the Icelandic State; the Research Fund of the University of Iceland; the Icelandic Science and Technology Research Programme for Postgenomic Biomedicine, Nanoscience and Nanotechnology; and the National Science Council of the Republic of China through Contract No. NSC97-2112-M-239003-MY3.

\section{References}

[1] C. W. J. Beenakker and H. van Houten, in Solid State Physics: Advances in Research and Applications, edited by H. Ehrenreich and D. Turnbull (Academic, New York, 1991), Vol. 44, pp. 1-228.

[2] F. E. Camino, W. Zhou, and V. J. Goldman, Phys. Rev. B 72 (2005) 155313.

[3] M. Sigrist, T. Ihn, K. Ensslin, M. Reinwald, and W. Wegscheider, Phys. Rev. Lett. 98 (2007) 036805.

[4] S. A. Gurvitz, Phys. Rev. B 51 (1995) 7123.

[5] R. Landauer, IBM J. 1 (1957) 223.

[6] M. Büttiker and R. Landauer, Phys. Rev. Lett. 49 (1982) 1739.

[7] M. Yang and S.-S. Li, Phys. Rev. B 70 (2004) 045318. 